\title{
Corrigendum
}

David Klemperer*, Ullrich Bauer, Robert Francke, Marie-Luise Dierks, Bernt-Peter Robra, Rolf Rosenbrock and Jürgen Windeler

\section{Corrigendum zu: Positionspapier zur Weiterent- wicklung der Gesundheitsversorgungsforschung und zu Themen für künftige Ausschreibungen von Forschungsvorhaben}

DOI: $10.1515 /$ pubhef-2015-1001

Corrigendum zu: David Klemperer, Ullrich Bauer, Robert Francke, Marie-Luise Dierks, Bernt-Peter Robra, Rolf Rosenbrock, Jürgen Windeler. März 2015. Positionspapier zur Weiterentwicklung der Gesundheitsversorgungsforschung und zu Themen für künftige Ausschreibungen von Forschungsvorhaben. Public Health Forum. Band 23, Heft 1, Seiten 47-50 (DOI: 10.1515/pubhef-2015-0019):

Auf der S. 49 ist in der rechten Spalte unter der Überschrift Präferenzsensitive Versorgung (preference- sensitive care) das Wort „Interventionen“ durch „Gesundheitsprobleme“ zu ersetzen. Die korrigierte Version lautet wie folgt:

\section{Präferenzsensitive Versorgung (preference-sensitive care)}

bezieht sich auf Gesundheitsprobleme, für die sich aus der Evidenz mehr als eine vernünftige Lösung ergibt, häufig einschließlich der Möglichkeit der Nicht-Behandlung.

*Korrespondenz: David Klemperer, Ostbayerische Technische Hochschule Regensburg, Fakultät für Sozial- und Gesundheitswissenschaften, Seybothstraße 2, 93053 Regensburg, Deutschland, E-mail: david.klemperer@hs-regensburg.de Ullrich Bauer: Universität Bielefeld, Fakultät für Bildungswissenschaften, Universitätsstraße 25, D-33615 Bielefeld, Deutschland

Robert Francke: Universität Bremen, Fachbereich Rechtswissenschaft, Universitätsallee, GW1, 28359 Bremen, Deutschland

Marie-Luise Dierks: Institut für Epidemiologie, Sozialmedizin und Gesundheitssystemforschung, Medizinische Hochschule Hannover, OE 5410, Carl-Neuberg-Str. 1, 30625 Hannover, Deutschland Bernt-Peter Robra: Klinikum der Otto-von-Guericke-Universität Magdeburg Institut für Sozialmedizin und Gesundheitsökonomie (ISMG), Leipziger Str. 44, 39120 Magdeburg, Deutschland Rolf Rosenbrock: Deutscher Paritätischer Wohlfahrtsverband - Gesamtverband e. V., Oranienburger Str. 13-14, D-10178 Berlin, Deutschland Jürgen Windeler: Institut für Qualität und Wirtschaftlichkeit im Gesundheitswesen, Im Mediapark 8 (KölnTurm), 50670 Köln, Deutschland 